\title{
Sensitivity analysis in the technical potential assessment of onshore wind and ground solar photovoltaic power resources at regional scale
}

\author{
Arthur Bossavy $^{1}$, Robin Girard ${ }^{2}$, and George Kariniotakis ${ }^{3}$ \\ ${ }^{1,2,3}$ MINES ParisTech, PSL - Research University,, PERSEE - Center for Processes, \\ Renewable Energies \& Energy Systems,, CS 10207 rue Claude Daunesse, 06904 Sophia \\ Antipolis Cedex, France.
}

This is a pre-print version. Please refer to as: Bossavy et al. Sensitivity analysis in the technical potential assessment of onshore wind and ground solar photovoltaic power resources at regional scale. Applied Energy. 2016. Vol. 182, p. 145-153

Corresponding author email: abossavy@gmail.com

\begin{abstract}
Potential assessment has served various objectives in the development of renewable energies. However, the prospective nature of this type of assessment sometimes makes it difficult to evaluate and compare estimation results based on different data and modeling. To facilitate this comparison, uncertainty estimates need to be systematically provided. Since potential assessment sometimes relies on numerous parameters, this may first require determining the most important inputs to focus on. In this paper, we propose a sensitivity analysis methodology based on Sobol indices so as to identify the main inputs from a nonlinear assessment model. We illustrate the proposed methodology through analyzing sensitivity in an onshore wind and ground solar photovoltaic (PV) potential assessment covering two French regions. As a result, we show that, when estimating the potential of these renewable energy sources, parameters defining surface availability are more prevalent than those related to technology.
\end{abstract}




\section{Nomenclature}

\begin{tabular}{|c|c|}
\hline & Parameters involved in wind power production simulation \\
\hline$h_{\mathrm{wp}_{n}}$ & $\begin{array}{l}\text { capacity factor increase ratio from the use of new wind turbine technol- } \\
\text { ogy, }\end{array}$ \\
\hline \multirow{2}{*}{$w_{\mathrm{wp}_{n}}$} & proportion of new wind turbine technology installed capacity. \\
\hline & Parameters involved in solar PV production simulation \\
\hline$h_{\mathrm{pv}}$ & $\begin{array}{l}\text { capacity factor increase ratio from improving polycristallin, thin film } \\
\text { (i.e. amorphous silicon) and monocristallin PV module technologies, }\end{array}$ \\
\hline$\left(w_{p}, w_{a}\right)$ & $\begin{array}{l}\text { installed capacity proportions of polycristallin and thin film technologies } \\
\text { among those with fixed orientation }\left(1-w_{p}-w_{a} \text { being the proportion of }\right. \\
\text { monocristallin technology), }\end{array}$ \\
\hline$(\alpha, \beta)$ & tilt and orientation of PV power plants with fixed orientation, \\
\hline \multirow[t]{2}{*}{$\left(w_{t_{1}}, w_{t_{2}}\right)$} & $\begin{array}{l}\text { installed capacity proportions of } \mathrm{PV} \text { power plants with single-axis or } \\
\text { dual-axis tracking systems }\left(1-w_{t_{1}}-w_{t_{2}} \text { being the proportion of power }\right. \\
\text { plants with fixed orientation). }\end{array}$ \\
\hline & Parameters involved in power plants' implantation strategy \\
\hline P.a. & $\begin{array}{l}\text { binary parameter indicating whether or not protected natural areas are } \\
\text { constraints to wind and solar power capacity implantation that can be } \\
\text { alleviated, }\end{array}$ \\
\hline R.A.t.l. & $\begin{array}{l}\text { binary parameter indicating whether or not radars and military air traffic } \\
\text { lanes are constraints to wind power capacity implantation that can be } \\
\text { alleviated, }\end{array}$ \\
\hline alt $_{\max }$ & maximum altitude suitable for plants' installation, \\
\hline$\nabla$ alt $\max$ & maximum altitude gradient suitable for plants' installation, \\
\hline$\left(\nabla\right.$ alt $\left.\max _{\text {maxth }}^{\text {north }}\right)$ & $\begin{array}{l}\text { critical relief conditions in north orientation for ground PV plants' in- } \\
\text { stallation, }\end{array}$ \\
\hline$\left(r_{l c}\right)_{l c}$ & acceptability ratios defined by type of surface $l c$ \\
\hline$c_{\min }$ & minimum capacity factor suitable for plants' installation, \\
\hline$c_{u}$ & installed capacity by surface unit at power plant scale. \\
\hline
\end{tabular}




\section{Introduction}

Potential assessment has served various objectives in the development of renewable energies. Designed to cover a large geographical scale (e.g. global or continental), it provides broad estimates of the technical feasibility of satisfying global consumption with sufficient production [1, 2, 3, 4, its cost [5, 6], and the associated climate change mitigation potential [7. Potential assessment has been devised to guide energy policies for decades to come and should therefore integrate any anticipated (e.g. societal) changes likely to affect the development of power source alternatives at global level [5]. On the other hand, at a local (e.g. city or plant) scale, potential assessment supports estimations of the expected returns on investment of prospective-phase industrial projects. It can rely on highly detailed modeling thanks to on-site measurements employing the most recent instrumentation technology, such as LIDAR [8, 9].

The increasing availability of numerical data has resulted in considerable research to develop potential assessment methodologies based on GIS tools [10, 11, 12, 8, 13. Despite consensus on the definition of power source potential and the generalized use of GIS-tools, it is difficult to evaluate and compare the results established by various studies. This is because, modeling is highly dependent on geographical scale and may vary significantly depending on the data available, their resolution or numerical representation. Moreover, the prospective nature of these studies makes it impossible to evaluate and compare ex-post their respective methodologies ${ }^{2}$

Another major limitation often observed in potential assessment studies is the low number of results regarding uncertainty estimation ${ }^{3}$ Some exceptions are noteworthy. In [14, a methodology is developed to estimate the technica ${ }^{4}$ potential of rooftop solar photovoltaic (PV) at regional scale (exemplified through the autonomous Spanish regions). The available roof surface is estimated for different urban area typologies. For each typology, this estimation is made from a minimum sample of municipalities ensuring a predefined confidence leve ${ }^{5}$ Uncertainty about available roof surface is then translated into solar potential uncertainty estimations. In [6], analytic density functions are proposed to characterize the distribution of renewable power source sites' productivity. Such analytic functions are then used to derive closed-forms of cost-supply curve ${ }^{6}$ in the global economic potential evaluation of various renewable energy sources. The analytical form of cost-supply curves leads to a straightforward deduction of associated uncertainty estimates.

The methodology introduced in 6 allows to derive economic potential uncertainty estimates from technical potential uncertainty estimates. Thus, the reliability of the former is closely related to, while not relying on, assumptions made in deriving the latter. To that matter, assuming such an uncertainty is driven by a single parameter (such as available rooftop surface in [14]), may seem insufficient ${ }^{7}$. Indeed, potential assessment generally depends on numerous parameters whose influence on resource estimation relies on complex nonlinear relationships. On the other hand, systematically deriving uncertainty estimates from either parametric modeling or Monte-Carlo simulations may require to focus on one or just a few prevailing parameters. Therefore, such parameters need to be first rigorously identified using an appropriate and reproducible methodology.

In this paper, we propose a methodology to rank the influence of parameters in potential assessment. More precisely, we propose a sensitivity analysis to evaluate the relative influence of each input parameter on output estimation variability. We use Sobol indices whose computation is derived from Monte-Carlo simulations. Using such indices has the great advantage of keeping model assumptions to a minimum. In

\footnotetext{
${ }^{1}$ Geographic Information System.

${ }^{2}$ Unless the considered case study areas end up fully exploiting a given power source in the future.

${ }^{3}$ Of course part of this uncertainty is intrinsic to the methodology employed and, as we point out, cannot be rigorously evaluated.

${ }^{4}$ Based on estimations of primary energy sources in a given area, it generally integrates geographical constraints into installing power plants and the technological characteristics of energy-conversion devices. Integrating economic or social constraints into further estimations leads to evaluating the so-called economic potential.

${ }^{5}$ Associated with some parametric assumptions unfortunately not discussed in the paper.

${ }^{6}$ A cost-supply curve (more precisely its inverse function) gives the energy potential as a function of the maximum acceptable marginal extraction cost.

${ }^{7}$ For some renewable power sources, such as PV or wind power, available surface is generally accepted as one of the key parameter driving potential uncertainty [6, 15].
} 
particular, their computation requires no (e.g. linear) assumption on the resource estimation modeling function. Monte-Carlo simulations and Bayesian approaches have already been proposed in the literature to evaluate (wind) power potential at local scale [16, 17. To our knowledge, this is the first time it has been used to conduct a proper sensitivity analysis in potential estimation. We illustrate the proposed approach through evaluating the technical potential of both onshore wind and ground solar PV in two French regions.

The paper is organized as follows: in Section 2, we describe the data and modeling used in wind and solar PV power production simulation. We also describe the geographical constraints and implantation strategies of power plants that are used to evaluate wind and solar power potential. In Section 3 , we give a brief description of the Sobol indices and the sensitivity analysis methodology. In Section 4 , we describe the case study used to illustrate our approach. In particular, we describe the parametric assumption used for parameter sampling. The results of our simulations are presented and discussed in Section 5 . Some conclusions and perspectives of our work are given in Section 6 .

\section{Data and modeling for wind and solar power potential assess- ment}

In this section, we describe the data and modeling used to estimate renewable energy potential. The methodology used can be summarized by three steps:

1. First, sites' normalized productivity (i.e. capacity factor) is estimated at any given location. The estimation relies on renewable production simulations derived from meteorological data. Models involved in such simulations are described in 2.1 and 2.2 .

2. Next, geographical constraints are gathered to define zones in which power plants' implantation is considered impossible. These constraints are detailed in Section 2.3.1.

3. Finally, power capacities are assumed to be installed in the remaining areas based on (highest) capacity factors, and the potential is estimated. This final step is described in 2.3 .2

\subsection{Data and modeling for wind power production simulation}

\subsubsection{Wind speed data and extrapolation to turbine's hub height}

In our work, wind power simulations rely on wind speed estimates derived by combining two meteorological reanalysis datasets. We used ECMWF 8 wind speed reanalysis data (time resolution: $6 \mathrm{~h}$, spatial resolution: $10 \mathrm{~km} \times 14 \mathrm{~km}$ ) to spatially interpolate MERRA ${ }^{9}$ data (time resolution: 1h, spatial resolution: $50 \mathrm{~km} \times 55 \mathrm{~km}$ ). To do so, we employed a time-adaptive statistical linear model (see [18, Section 1.1.1, for more details). Initially provided at a $10 \mathrm{~m}$ altitude, wind speed data was then extrapolated to an $80 \mathrm{~m}$ altitude using a logarithmic profile (see [1, Equation (1)), with a fixed surface roughness value ${ }^{10}$ of 0.05. Such a simplification associated with an approximate choice of the turbine's hub height would normally be highly detrimental to estimating power accurately. However, in our approach, wind speed data is automatically recalibrated later on, during the power conversion proces:

\subsubsection{Statistical regional power curve model}

To convert wind speed into power, we used a statistical model. For calibration purposes, we disposed of regional production data covering one year of production (2013) provided by the French TSO The

\footnotetext{
${ }^{8}$ European Center for Medium Range Weather Forecasts, http://www.ecmwf.int.

${ }^{9}$ Modern Era-Retrospective analysis for Research and Applications, from NASA, http://gmao.gsfc.nasa.gov.

${ }^{10}$ Somewhat intermediate value corresponding to arable land, see http://www.dataforwind.com for more details.

${ }^{11}$ We use a statistical power curve model, which allowed us to terminate wind speed recalibration before power conversion. This is common practice, for instance, in short-term wind power forecasting [19].

${ }^{12}$ Transmission System Operator: Reseau de Transport d'Electricite, http://www.rte-france.com.
} 
proposed model is a piecewise model inferring regional production from average wind speed estimates throughout the region, weighted by the installed capacity at each (meteorological) pixel. First, power linearly increases with wind speed for low wind speed values (i.e. $v \leq 3 \mathrm{~m} . \mathrm{s}^{-1}$ ), then according to a smoothing spline model $\left(3 \mathrm{~m} \cdot \mathrm{s}^{-1} \leq v \leq 10.5 \mathrm{~m} . \mathrm{s}^{-1}\right)$, and then power plateaus, before linearly decreasing with very high wind speeds $\left(20 \mathrm{~m} . \mathrm{s}^{-1} \leq v \leq 24 \mathrm{~m} . \mathrm{s}^{-1}\right)$ until complete shutdown (see [18], Section 1.1.2, for more details).

\subsubsection{Improvements in wind turbine technology}

A site's productivity is defined by its long-term average production-to-nominal-capacity ratio, the socalled capacity factor $c_{\mathrm{wp}}=\mathbb{E}\left[p_{t}\right]$ ( $p_{t}$ being the production expressed in $\%$ of the nominal capacity $\left.P_{n}\right)$. It was computed from hourly simulations covering a 9-year period ${ }^{13}$ (2005-2013). In the near future, the evolution of wind turbine technology should result in sites with higher capacity factors despite relatively low winds [21, 22]. Taking such improvement into account, we chose to model the average capacity factor over a given area (i.e. a meteorological pixel), based on initial estimations, as follows:

$$
c_{\mathrm{wp}}= \begin{cases}\left(1+w_{\mathrm{wp}_{n}} h_{\mathrm{wp}_{n}}\right) c_{\mathrm{wp}} & \text { if } c_{\mathrm{wp}} \geq c_{\mathrm{min}} \\ \left(1+\left(w_{\mathrm{wp}_{n}}+0.5\right) h_{\mathrm{wp}_{n}}\right) c_{\mathrm{wp}} & \text { otherwise }\end{cases}
$$

where $h_{\mathrm{wp}_{n}}$ is the assumed capacity factor increase ratio attained thanks to new turbine technology and $w_{\mathrm{wp}_{n}}$ the proportion of installed capacity. We assume that this proportion depends on the average productivity of the usual technology over a pixel. If the latter is high, i.e. higher than a minimum acceptable capacity factor $c_{\min }$, then $w_{\mathrm{wp}_{n}} \in[0,0.5]$. Otherwise, the proportion of new turbine technology capacity is increased to $w_{\mathrm{wp}_{n}}+0.5$. This modeling reflects the fact that new turbine technology is dedicated to sites with low winds, i.e. characterized by low capacity factors of current technology.

\subsection{Data and modeling for solar power production simulation}

\subsubsection{Global horizontal irradiance data and projection on a tilted plane}

Global horizontal irradiance data was taken from the Helioclim 3 database [23, provided every 15min (then hourly averaged) at a spatial resolution of $5 \mathrm{~km} \times 7 \mathrm{~km}$. To estimate the level of irradiance on a tilted plane (i.e. the PV panels' orientation), we used the Muneer model [24]. This requires decomposing global irradiance into direct and diffuse components. To achieve this, we used the model proposed in 25]. Solar angles, TOA ${ }^{14}$ irradiance and optical air mass estimates used throughout this process were computed using the "solar_geometry" C library ${ }^{15}$ For more information on the parameterization of these models, refer to [18] (Section 1.2.1).

\subsubsection{Power conversion modeling}

Converting solar irradiance into electrical power requires PV modules and inverter modeling. PV modules' efficiency depends on the irradiance level and is based on performance measurements from technologies available on the market. We also introduced a thermal modeling of the modules' efficiency depending on the cells' temperature. This allows us to distinguish between various technologies (monocristallin, polycristallin, thin film and concentrated PV, i.e. CPV), taking their installation set-up into account. We also used a performance assessment of market technologies to derive the efficiency of inverters depending on the load factor. Other losses, before (i.e. DC losses) and after (i.e. AC losses) the inverters, were also taken into account. For more information, refer to [18] (Section 1.2.2 and 1.2.3).

\footnotetext{
13 Long-term climatic variations, such as NAO (North Atlantic Oscillations), make it difficult to rely on capacity factors estimated from such a short time period [20]. Considering such an uncertainty in potential assessment is nevertheless beyond the scope of this study.

${ }^{14}$ Top-of-atmosphere.

${ }^{15}$ Developed at the Observation Impact Energy Center of MINES ParisTech, http://www.oie.mines-paristech.fr.
} 


\subsubsection{Technology mix and improvements}

We introduced a technology mix and improvements by modeling the capacity factor. Let $c_{m}, c_{p}, c_{a}, c_{t_{1}}$ and $c_{t_{2}}$ denote the capacity factors associated with monocristallin, polycristallin, thin film, single-axis CPV and dual-axis CPV technologies respectively. Similarly, we introduced the installed capacity proportions $w_{p}, w_{a}, w_{t_{1}}, w_{t_{2}}$. Then, we modeled the average ground PV capacity factor as:

$$
c_{\mathrm{pv}}=w_{t_{1}} c_{t_{1}}+w_{t_{2}} c_{t_{2}}+\left(1-w_{t_{1}}-w_{t_{2}}\right)\left(1+h_{\mathrm{pv}}\right)\left(w_{p} c_{p}+w_{a} c_{a}+\left(1-w_{p}-w_{a}\right) c_{m}\right)
$$

where $h_{\mathrm{pv}}$ is the assumed capacity factor increase ratio that can be expected in the future thanks technology improvements in PV modules[26]. In the absence of additional information, we chose not to extend such improvements to CPV technology.

\subsection{Geographical constraints and potential assessment methodology}

\subsubsection{Geographical constraints}

Once capacity factors had been estimated, the next step was to exclude locations unavailable for power plant installation. In our study, four constraints prohibiting power plant deployment were identified and taken into account. Land may be judged unavailable due to:

- Unsuitable soil type or conflicting land usage

- Unsuitable landscape

- Protected natural areas

- Proximity to radars or military air traffic lanes (for wind only)

Surface types and land uses were determined using the Corine Land Cover 2006 database (CLC 2006, resolution: 100m). Urban and submerged territories were discarded ${ }^{16}$ The land types finally retained were technology-specific and are fully described in [18] (Section 1.3.1). Unsuitable landscapes are defined as locations that are at high altitudes (higher than alt $\max$ ), or too steep (altitude gradient higher than $\nabla$ alt $t_{\max }$ ), or with presumed inappropriate sun-exposure (for ground PV only: north-oriented $+/-\beta^{\text {northo }}$ altitude gradient higher than $\nabla$ alt $t_{\max } / 2$ ). The altitude data was taken from the IGN ${ }^{17}$ institute and has a $25 \mathrm{~m}$ spatial resolution. Protected natural areas were determined by the DREAL ${ }^{18}$ The locations of radars and military air traffic lanes were established using data from the French meteorological office MeteoFrance and Altech respectively.

\subsubsection{Potential assessment methodology}

To estimate the technical potential, we first estimated the surface $\mathcal{S}$ that can be allocated to production capacities based on the available (i.e. not constrained) surface $\tilde{\mathcal{S}}$. The estimation of $\tilde{\mathcal{S}}$ can be refined so as to only incorporate sites achieving minimum productivity ${ }^{19}$ i.e. a minimum capacity factor $c_{\min }$. We may then specify the notations: $\tilde{\mathcal{S}}=\tilde{\mathcal{S}}\left(c \geq c_{\min }\right), c$ denoting the capacity factor (i.e. $c_{\mathrm{wp}}$ or $c_{\mathrm{pv}}$, resp. in Equations (1) and (2)). Finally, $\mathcal{S}$ is defined as the minimum between $\tilde{\mathcal{S}}\left(c \geq c_{\min }\right)$ and some maximum usable (acceptable) proportion $r$ of the available surface regardless of productivity, i.e.:

$$
\mathcal{S}=\min \left\{r \tilde{\mathcal{S}}(c \geq 0), \tilde{\mathcal{S}}\left(c \geq c_{\min }\right)\right\} .
$$

\footnotetext{
${ }^{16}$ As in [27, a 500m-wide area around urban territories was systematically discarded in line with French legislation.

${ }^{17}$ Institut de l'Information Géographique et Forestière, http://www.ign.fr.

${ }^{18}$ Direction Régionale de l'Environnement, de l'Aménagement et du Logement, see for instance: http://www.paca.developpement-durable.gouv.fr.

${ }^{19}$ This can be regarded as the consideration of economic limitations since the profitability of renewable power plants is mostly determined from the capacity factor (see 28] for instance).
} 
The role of $r$ is to incorporate potential constraints that could not be explicitly modeled and integrated otherwise (such as population acceptance). The "acceptable" surface it defines (i.e. $r \tilde{\mathcal{S}}(c \geq 0)$ ) cannot be located a priori. Thus, one important hypothesis assumed in Equation (3) is that productive surfaces and those judged as "acceptable" come into the same geographical area (thus resulting in optimistically high surface estimations).

The potential in terms of installed capacity can be straightforwardly derived from (3) by multiplying $\mathcal{S}$ with the capacity that can be installed per surface unit at power plant scale ${ }^{20} c_{u}$. The energy potential $\mathcal{E}$ is computed assuming that capacities can be installed in the area $\Lambda$ of surface $\mathcal{S}$ with the most productive sites:

$$
\mathcal{E}=c_{u} \times \sum_{s \in \Lambda} c(s) \times 8760
$$

The estimation algorithm described above is applied to each land cover type after splitting the considered area into several subareas ${ }^{21}$ and the results are then added together. This brings two benefits. First, it allows us to discriminate acceptability ratio values depending on the surface type (thus changing the notation from $r$ to $r_{l c}, l c$ denoting the surface type. See [18], Section 1.3.1). Second, applying the algorithm to subdivisions of a (large) area avoids concentrating too much capacity in a single subarea with productive sites 22

\section{Methodology for the sensitivity analysis}

\subsection{Sobol indices definition}

To quantify the sensitivity of the resource estimation with respect to the various inputs considered in our model, we chose to use Sobol indices [29. These are globa ${ }^{23}$ sensitivity indices designed to estimate the relative share of ouput variance determined by the considered inputs or their interactions. Sobol indices are highly popular because their computation does not rely on any (e.g. linear) hypothesis about the underlying model function $f$ that links inputs $X_{i}, i \in \mathcal{I}:=\{1, \ldots, p\}$ to the output $Y=f\left(X_{\mathcal{I}}\right)$. However, it assumes that inputs $X_{i}$ are uncorrelated. Therefore, the following generic decomposition also has uncorrelated terms:

$$
Y=\mathbb{E}(Y)+\sum_{I \subseteq \mathcal{I}} f_{I}\left(X_{I}\right), \text { with } f_{I}\left(X_{I}\right)=\mathbb{E}\left[Y \mid X_{I}\right]-\sum_{I^{\prime} \subsetneq I} f_{I^{\prime}}\left(X_{I^{\prime}}\right)
$$

which allows us to decompose the variance $V$ of $Y$ the same way. Normalizing by $V$ finally reveals the Sobol indices $S_{I}$ :

$$
1=\sum_{I \subseteq \mathcal{I}} S_{I}, \text { with } S_{I}=V_{I} / V \text { and } V_{I}=\operatorname{var}\left(f_{I}\left(X_{I}\right)\right)
$$

Various summary statistics based on Sobol indices may then be proposed. In [30], the total sensitivity of a system $S_{T_{i}}$ to a given parameter $X_{i}$ was defined by adding up all indices involving parameter $X_{i}$, i.e. $S_{T_{i}}=\sum_{I, i \in I} S_{I}$. Extending that idea, it could be interesting to evaluate the total sensitivity of a system $S_{T_{L}}$ to some general aspect (e.g. technological characteristics, relief constraints, etc) whose definition potentially involves several parameters $L \subseteq \mathcal{I}$, i.e.

$$
S_{T_{L}}=\sum_{I, I \cap L \neq \emptyset} S_{I}
$$

\footnotetext{
${ }^{20}$ See 18 , Section 1.3.2, for more details on its estimation from the technology mix.

${ }^{21}$ In our study, French regions are subdivided into smaller administrative entities called départements.

${ }^{22}$ This would be unrealistic. Indeed, to benefit from smoothing that lowers renewable production variability and increases its predictability, in general the intention is to spatially distribute production capacities.

${ }^{23}$ Unlike using local methods, here the interest is to evaluate the global output variability considering that inputs vary in the entire inputs' space value and not just close to some particular value.
} 


\subsection{Sobol indices computation}

First, note that the definition of $f_{I}\left(X_{I}\right)$ in Equation (4) allows a recursive estimate of the Sobol indices, i.e.

$$
S_{I}=\frac{\operatorname{var}\left(\mathbb{E}\left[Y \mid X_{I}\right]\right)}{V}-\sum_{I^{\prime} \subsetneq I} S_{I^{\prime}} .
$$

Thus, to estimate $S_{I}$ considering that estimations of lower order indices are available, we simply estimate $\operatorname{var}\left(\mathbb{E}\left[Y \mid X_{I}\right]\right)$ (and $V$ ). This can be done through Monte Carlo simulations using two independent, equally sized, random samples of inputs: $\left(X_{\mathcal{I}}^{j, 1}\right)$ and $\left(X_{\mathcal{I}}^{j, 2}\right)$, where $j=1, \ldots, n[30$ :

$$
\begin{aligned}
& \hat{f}_{0}=\hat{\mathbb{E}}(Y)=\frac{1}{n} \sum_{j=1}^{n} f\left(X_{\mathcal{I}}^{j, 1}\right), \hat{V}=\frac{1}{n} \sum_{j=1}^{n} f^{2}\left(X_{\mathcal{I}}^{j, 1}\right)-\hat{f}_{0}^{2}, \\
& \text { and } \operatorname{vâr}\left(\mathbb{E}\left[Y \mid X_{I}\right]\right)=\frac{1}{n} \sum_{j=1}^{n} f\left(X_{\mathcal{I}}^{j, 1}\right) f\left(X_{I}^{j, 1}, X_{\bar{I}}^{j, 2}\right)-\hat{f}_{0}^{2},
\end{aligned}
$$

where $\bar{I}=\mathcal{I} \backslash I$. Estimating Sobol indices this way thus requires $(K+1) n$ evaluations of $f, K$ being the number of considered indices. Even when restricted to first order indices, this may still be too computationally demanding (especially when $p$ and $n$ are high and given that each evaluation of $f$ takes time). Thus, in our study, we used a single sample and the following heuristic to estimate partial variances based on cross products of $f$ :

$$
\operatorname{vâr}\left(\mathbb{E}\left[Y \mid X_{I}\right]\right)=\frac{1}{n} \sum_{j=1}^{n} f\left(X_{\mathcal{I}}^{j}\right) f\left(X_{\mathcal{I}}^{j^{\prime}}\right)-\hat{f}_{0}^{2},
$$

where $j^{\prime}$ is uniformly sampled from $\left\{k \neq j ; X_{I}^{k}, X_{I}^{j} \in B_{I}(j)\right\}$ and $B_{I}(j)$ has been defined a priori from observation binning (see [18, Section 2.1 for more details).

Using the proposed approach, the estimation of Sobol indices only requires $n$ evaluations of $f$. On the other hand, because dealing with high order indices would involve high dimensional bins, most likely with too few observations, it is preferable here to keep low (e.g. first) order indices. Moreover, we decided to repeat the estimation scheme so as to be able to derive average results and uncertainty estimates (different index estimates can be obtained through independent samplings of $j^{\prime}$ as defined above).

\section{Case Study}

\subsection{The considered areas}

The considered case-study areas are the French regions of Brittany and Provence-Alpes-Cote d'Azur (PACA). Respectively located in the north-west and south-east of France, these are distant regions characterized by very different climatic conditions, vegetation and landscapes. Britany is a flat region swept by strong winds and thus with high wind power potential a priori. In contrast, PACA is mountainous because of the Alps massif and enjoys from the highest sun exposure in France. Graphs detailing the considered case study areas are represented in Figure 1 .

\subsection{Distribution hypothesis for parameter sampling}

Here we describe the distribution hypothesis from which the various parameters were sampled. Unless specified, all parameters are assumed to be independent. The characteristics of the distribution hypothesis, along with comments justifying our choice, are given in Table1. In our study, the computation of Sobol indices relies on 10000 simulations of each parameter (and thus, on 10000 power resource estimations). Because of the computational burden, the simulation of some parameters relies on a bootstrap 
technique (see 31] for an introduction to bootstrap). We initially considered a smaller sample for these parameters. The final 10000 simulation sample was derived from a uniform sampling of the initial sampled values. We used this approach to limit to 500 the number of simulations associated with relief constraints computation. We also used it to limit to 200 the number of simulations of PV production time series considering the orientation of fixed panels.
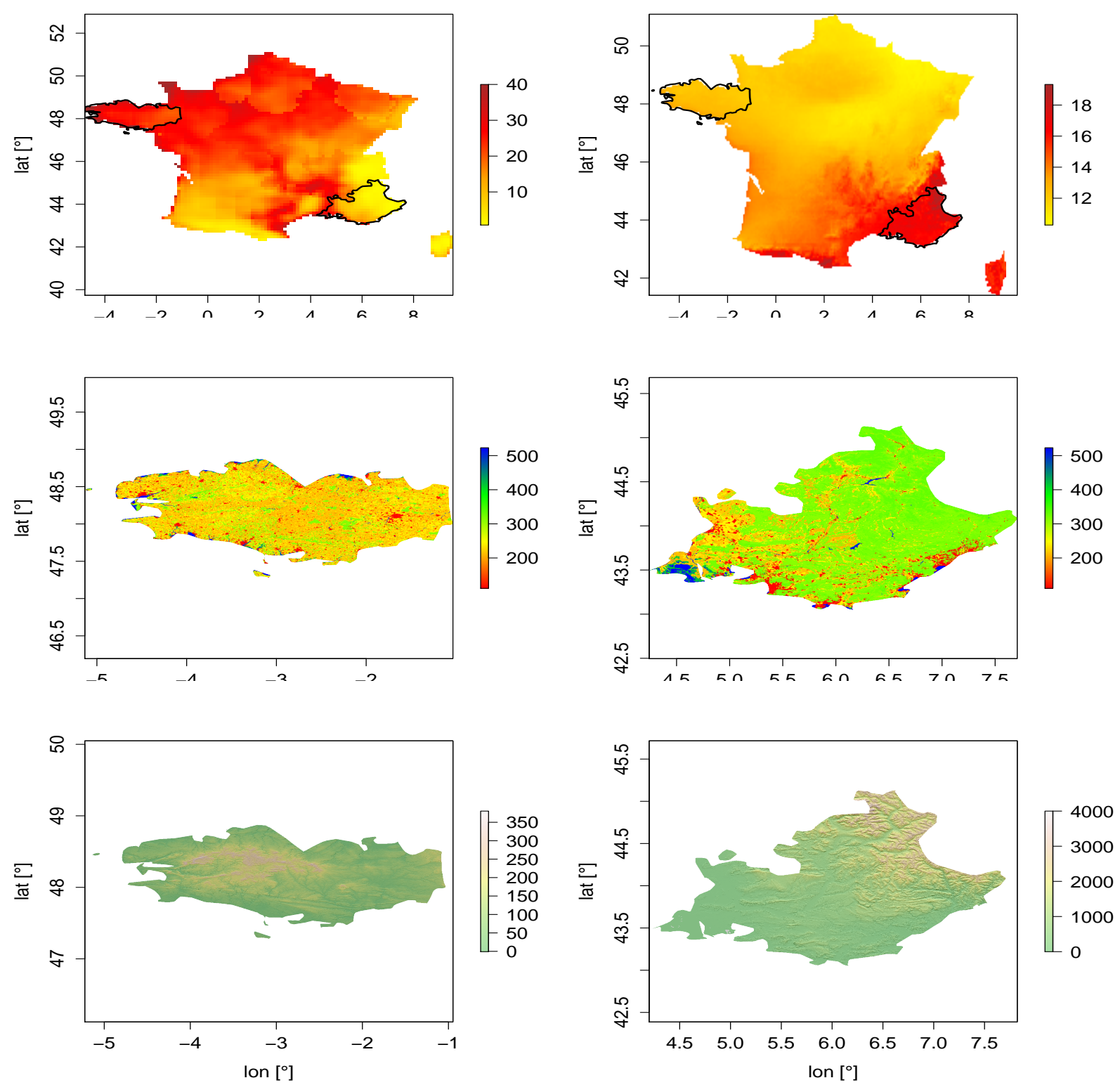

Figure 1: French region case study - Top: wind power (current technology) and PV (monocristallin, $\alpha=35^{\circ}, \beta=180^{\circ}$ ) capacity factors (in $\% P_{n}$ ) for the whole of France. The considered regions' boundaries are represented by polygons. Middle: surface type represented following the CLC 2006 database code (see [18, Section 1.3.1) for Brittany (left graph) and PACA (right graph). Bottom: altitude data (in m) from the IGN database. 
Table 1: Description of the distribution hypothesis from which power potential assessment parameters have been sampled. WP and PV are acronyms for Wind Power and Photovoltaic.

\begin{tabular}{|c|c|c|c|c|c|c|c|c|c|c|c|c|}
\hline & 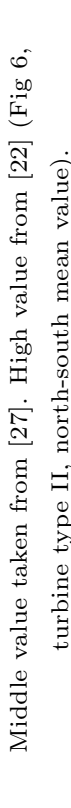 & 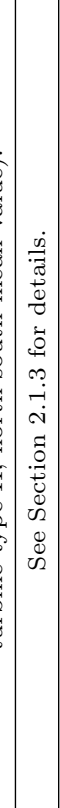 & 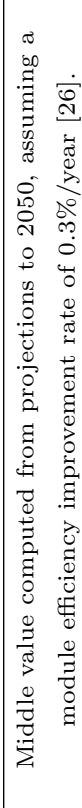 & 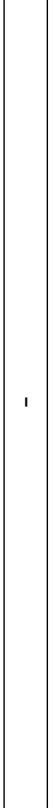 & & 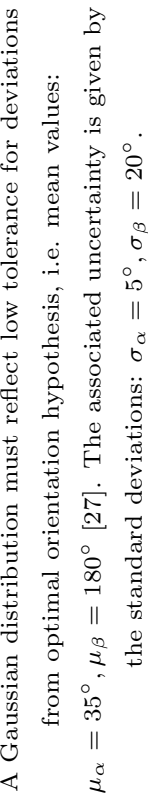 & & 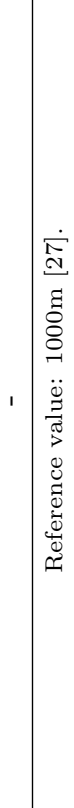 & 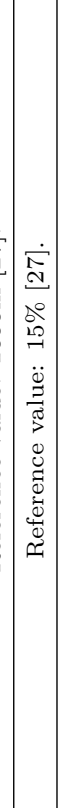 & 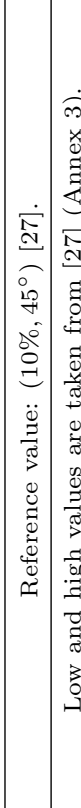 & 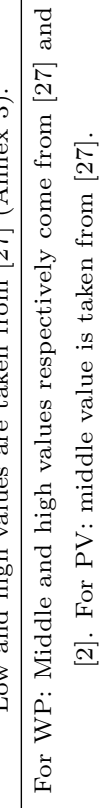 & 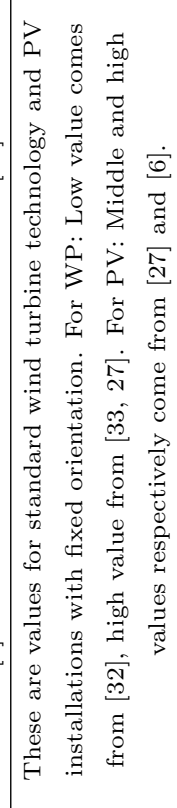 \\
\hline 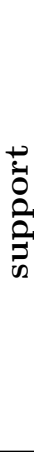 & 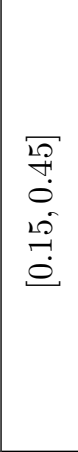 & $\begin{array}{l}10 \\
0 \\
0 \\
0 \\
0\end{array}$ & $\begin{array}{l}\tilde{r} \\
\tilde{O} \\
0 \\
0\end{array}$ & 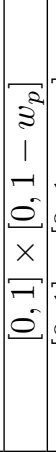 & 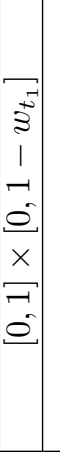 & 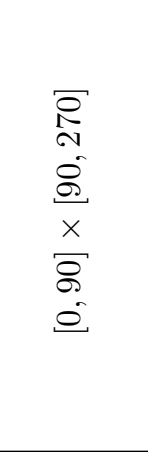 & $\begin{aligned} \Rightarrow \\
0 \\
0\end{aligned}$ & 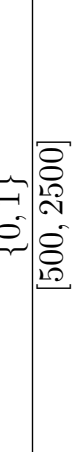 & $\left\{\begin{array}{l}\underset{9}{2} \\
20 \\
20\end{array}\right.$ & 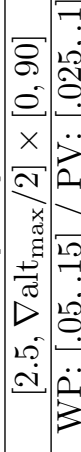 & 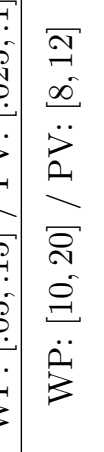 & 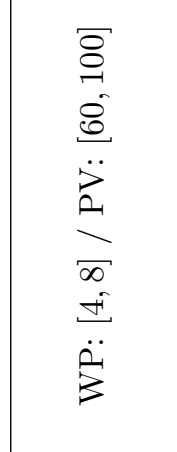 \\
\hline 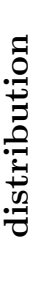 & 兘 & 苞 & 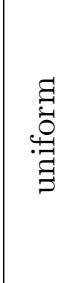 & 苛 & 离 & 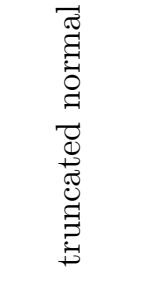 & : & 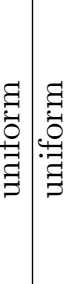 & 咅 & & 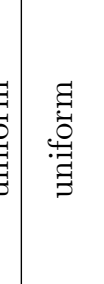 & 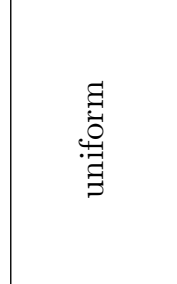 \\
\hline$\stackrel{\bullet}{\Xi}$ & ' & 1 & ' & 1 & ' & $\circ$ & & & $x^{\circ}$ & $\mid \begin{array}{ll}x & 1 \\
0 & 1 \\
0 & 1\end{array}$ & $\frac{R^{2}}{D^{\circ}}$ & $\frac{g}{\xi}$ \\
\hline 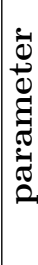 & $\stackrel{\text { ले }}{3}$ & इे & $\stackrel{2}{2}$ & కే & $\Xi$ & o & & 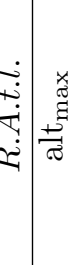 & 晃 & 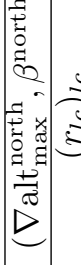 & हूँ & ن \\
\hline
\end{tabular}




\section{Results}

\subsection{Onshore wind}

Histograms representing the distribution of onshore wind resource estimation results are shown in Figure 2 Results are given in terms of the expected annual energy production density, for both Brittany (left graph) and PACA (right graph). In terms of energy production, the estimated resource mean value (about $30 \mathrm{TWh} / \mathrm{y}$ for Brittany and $5 \mathrm{TWh} / \mathrm{y}$ for PACA) is of the same order of magnitude ${ }^{24}$ as the results presented in 27. Brittany has a higher potential than PACA basically because of higher capacity factors and larger surface areas available for capacity deployment. The uncertainty associated with such an estimation is also lower in the case of Brittany. The standard deviation alone is not sufficient to make a fair comparison here and must be analyzed with respect to the order of magnitude of expected potential. For instance, the standard-deviation-to-mean ratio (namely the coefficient of variation) equals 0.32 for Brittany and is nearly twice as high (i.e. 0.57) for PACA.

\section{Brittany}

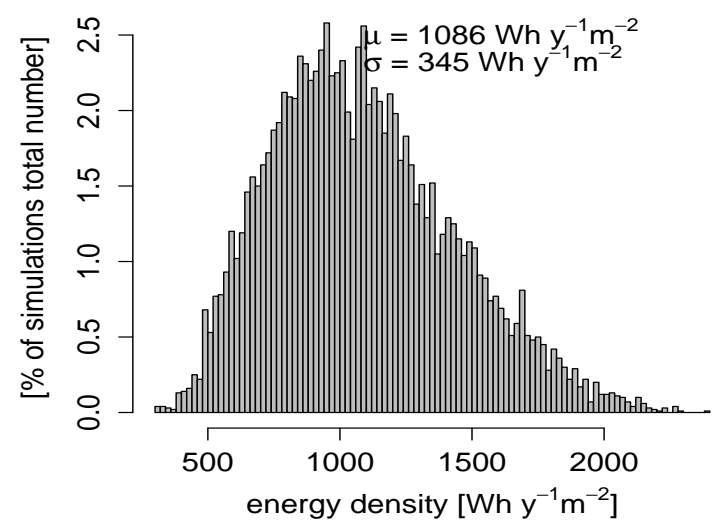

Provence-Alpes-Cote d'Azur

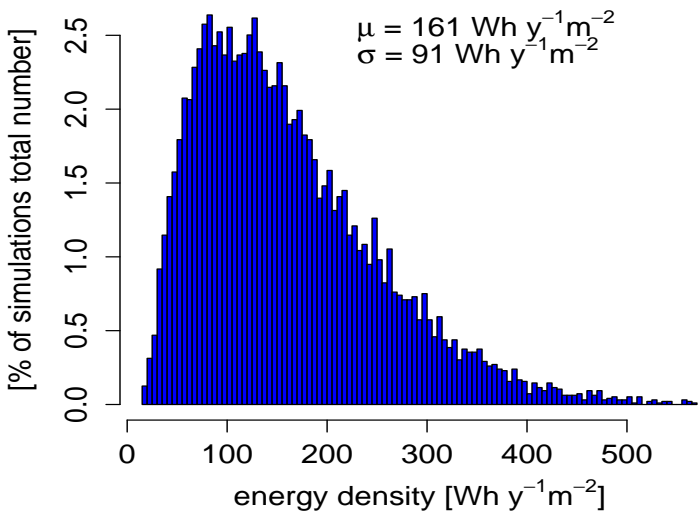

Figure 2: Distribution of the resource estimation results - Histograms representing the distribution of onshore wind energy resource estimation results. Results are given for both Brittany (left graph) and PACA (right graph). Mean estimation result $\mu$ and standard deviation $\sigma$ are also shown in the graphs.

First order Sobol indices are shown in Figure 3 , for both the Brittany and PACA regions. In this figure, mean estimation results with error bars (+/- 2 standard deviation) were computed based on 250 estimation replicates. As an initial remark, note that based on the proposed model and for the considered case study, all (in the case of Brittany) or most of the estimated resource variance is determined by input parameters alone.

In PACA, most of the resource sensitivity is determined by whether or not protected or forbidden areas are considered: $S_{P . a .} \simeq 41 \%$ and $S_{R . A . t . l .} \simeq 14 \%$. On the other hand, in Brittany the resource is particularly sensitive to acceptability ratios (especially on farming land: $S_{r_{l c_{a}}} \simeq 41 \%$, preponderant in that region, see Figure 1), defining available surfaces among those not discarded a priori.

Among other aspects related to surface availability, it is noteworthy that the resource in both regions is sensitive to discarded surfaces with steep relief: $S_{\nabla_{\text {alt }} \max } \simeq 10 \%$ for Brittany and $S_{\nabla_{\text {alt }} \text { max }} \simeq 13 \%$ for PACA. This is surprising considering that the lanbdscape in Brittany is relatively flat. This result may be due to the relatively harsh selection of suitable relief when $\nabla$ alt max $_{\text {is }}$ close to the chosen low limit

\footnotetext{
${ }^{24}$ Especially for Brittany. For PACA, mean estimation results are about twice as high here, both for onshore wind and ground PV power sources. This must be due to hypothesis variations in hypothesis associated with parameters defining surface availability. In particular, alt $\max$ and $\nabla$ alt max $_{\text {max }}\left(r_{l c}\right.$ also for $\left.\mathrm{PV}\right)$ have been set higher in average here and resources in PACA must be particularly sensitive to it.
} 


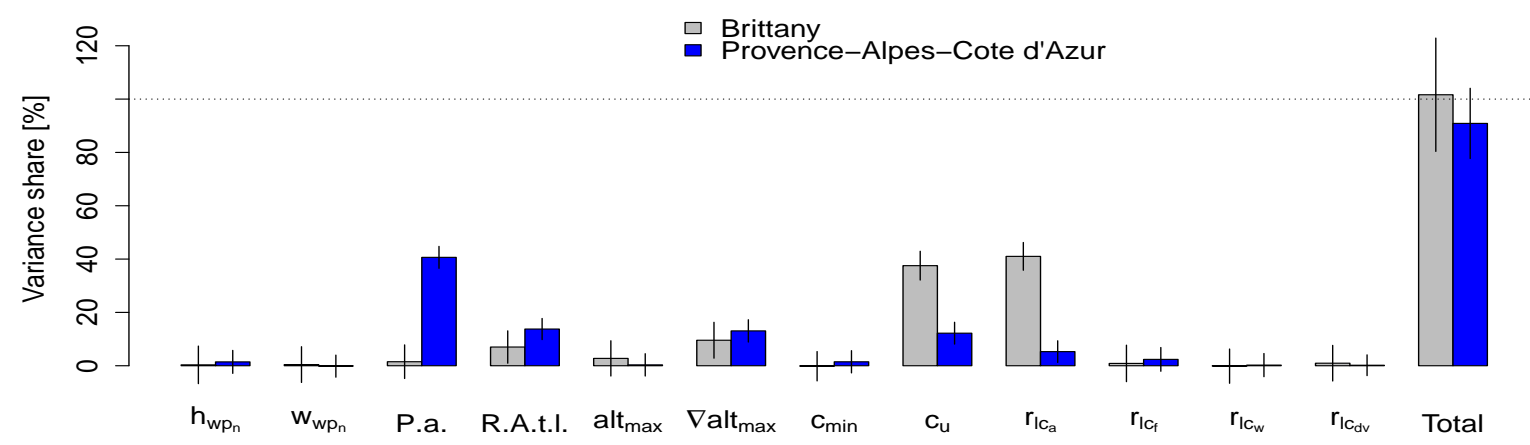

Figure 3: First order Sobol Indices - Barplots showing the relative share of wind energy resource estimation variance determined by input parameters alone (first order Sobol indices). The total relative share of output variance explained by the considered indices is also shown. Results are given for both the regions Brittany and PACA. Mean estimation results and error bars (+/- 2 standard deviation) were computed based on 250 estimation replicates.

value (i.e. $\nabla$ alt $\max =5 \%$ ). In future studies, efforts should be made to narrow the uncertainty range in which such a parameter lies in order to better appreciate output sensitivity.

In terms of technology, the results of our study show that the resource estimation may be highly sensitive to the installed capacity per surface unit at a power plant scale $c_{u}$. Indeed, the relative share of output variance explained by this parameter alone $S_{c_{u}}$ is about $38 \%$ for the Brittany case study, and about $12 \%$ for PACA. Beyond this parameter, we can determine the impact that turbines' nominal capacity and wind farm design may have on the resource. Considering the PACA case study, there may be interactions involving how sensitive the resource estimation is to parameter $c_{u}$ ( Total $=\sum_{i} S_{\{i\}}<100 \%$, see Figure 3). An association with the new turbine technology installed capacity ratio $w_{\mathrm{wp}_{n}}$ (allowing us to estimate the average value of $c_{u}$ for the technology mix, see [18, Section 1.3.2), may be considered as a natural candidate. However, further estimations showed that on average $S_{w_{\mathrm{wp}_{n}}, c_{u}} \simeq 0$.

Focusing on new turbine technology, its influence on resource estimation may be difficult to assess here. Indeed, in our model, the characterization of such a technology involves the parameter $c_{u}$ which is not exclusively related to it 25 . Moreover, it is probable that any influence such a technology may have on resource estimation actually comes through this parameter alone. Indeed, both parameters $h_{\mathrm{wp}_{n}}$ and $w_{\mathrm{wp}_{n}}$ that also participate in new turbine technology characterization seem to have no influence on their own, i.e. $S_{h_{\mathrm{wp}_{n}}} \simeq S_{w_{\mathrm{wp}_{n}}} \simeq 0$. For the PACA case study, further investigations showed no contribution from interactions as obvious candidates, i.e. $S_{w_{\mathrm{wp}_{n}}, c_{u}} \simeq 0$ (as already explained above), and $S_{h_{\mathrm{wp}_{n}}, w_{\mathrm{wp}_{n}}} \simeq 0$ (the latter interaction being involved in estimating the capacity factor from the technology mix, see Equation (1) Section 2.1.3.

\subsection{Ground PV}

Histograms representing the distribution of ground PV resource estimation results are shown in Figure 4. Results are given in terms of expected annual energy production density, for both the Brittany (left graph) and PACA (right graph) regions. The same asymmetric nature of the estimation results distribution can be observed here, as it was for wind power. While results tend to show higher potential for PACA this time, the uncertainty associated with such an estimation is still lower in the case of Brittany (the coefficient of variation being equal to 0.45 compared to 0.76 for PACA).

First order Sobol indices are shown in Figure 5 for both Brittany and PACA. While the wind energy

\footnotetext{
${ }^{25}$ Installed capacity by surface unit at power plant scale for new turbine technology has been defined as a fixed ratio, i.e. $2 / 3$, of that associated to standard technology (see also [18, Section 1.3.2).
} 


\section{Brittany}

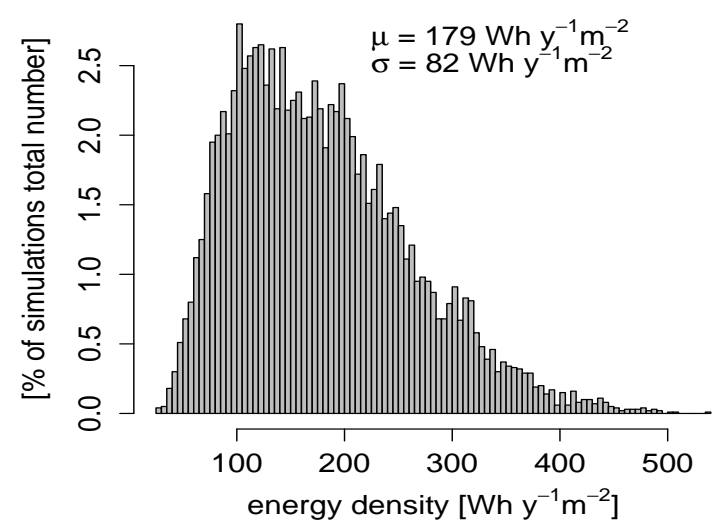

Provence-Alpes-Cote d'Azur

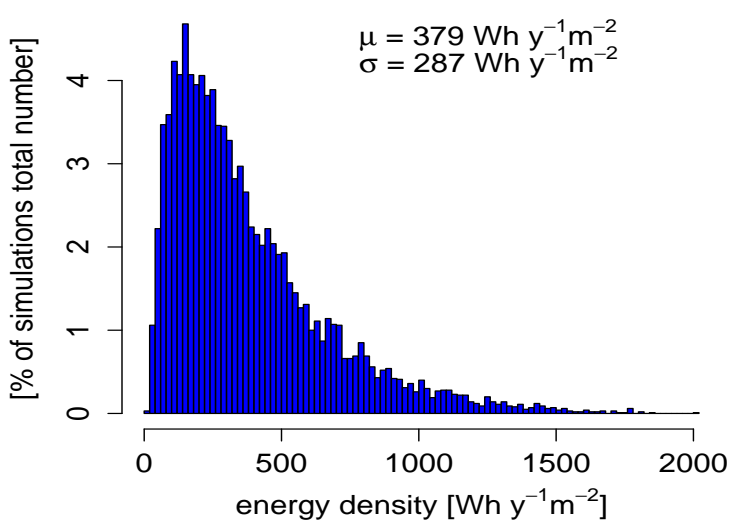

Figure 4: Distribution of the resource estimation results - Histograms representing the distribution of ground solar photovoltaic energy resource estimation results. Results are given for both Brittany (left graph) and PACA (right graph). The mean estimation result $\mu$ and standard deviation $\sigma$ are also shown in the graphs.

resource only appears to be slighlty sensitive to some parameter interactions for PACA, here the estimated resource variability seems to be systematically determined by input parameters alone.

Resource sensitivity to surface availability related aspects is stronger here than it was for onshore wind. About $85 \%$ and $95 \%$ of output variance is determined by the associated parameters $L:=$ $\left\{P . a .\right.$, alt $_{\max }, \nabla$ alt $_{\max }, \nabla$ alt max $\left.\left._{\text {max }}, \beta^{\text {north }}, r_{l c}\right\}\right\}^{26}$ for Brittany and PACA respectively. As for onshore wind, the PV resource in PACA is more sensitive to the consideration of protected areas and unsuitable landscapes (i.e. $S_{T_{L \backslash\left\{r_{l c}\right\}}} \simeq 73 \%$ ), while in Brittany the resource is more sensitive to acceptability ratios defining the remaining available surfaces (i.e. $S_{r_{l c}} \simeq 57 \%$ ).

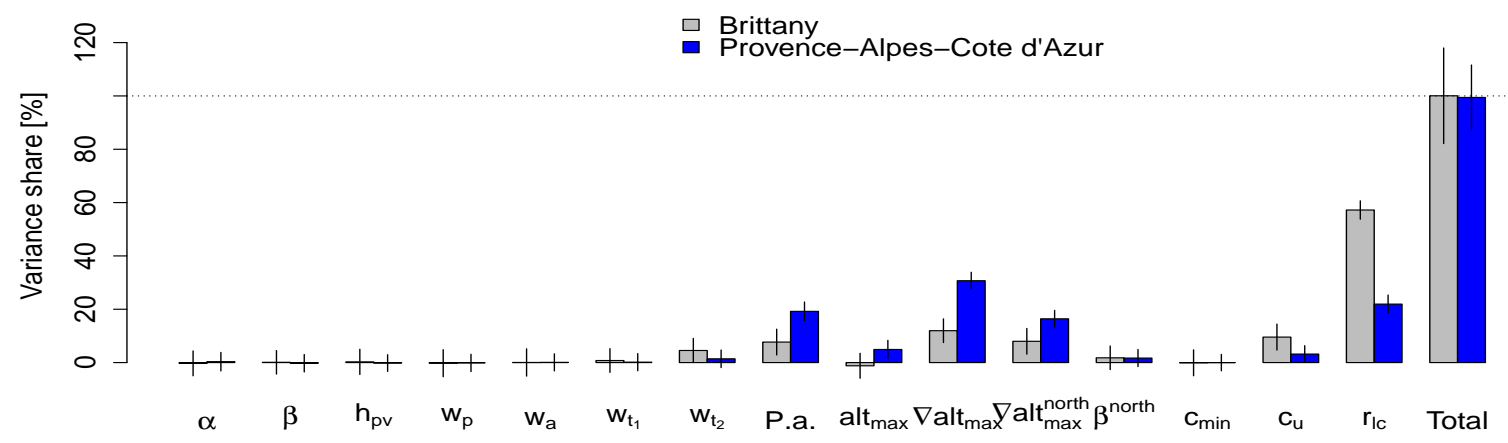

Figure 5: First order Sobol Indices - Barplots showing the relative share of ground PV resource estimation variance determined by input parameters alone (first order Sobol indices). The total relative share of output variance explained by the considered indices is also shown. Results are given for both the regions Brittany and PACA. Mean estimation results and error bars $(+/-2$ standard deviation) were computed based on 250 estimation replicates.

\footnotetext{
${ }^{26}$ For PV, to keep the number of parameters reasonable, we decided not to distinguish between the different land types when setting the acceptability ratio value.
} 
Focusing on the technological aspect, the main parameter to which the resource estimation is sensitive is the installed capacity per surface unit at power plant scale $c_{u}$. This sensitivity is nevertheless about four times lower than it was for onshore wind ( $S_{c_{u}} \simeq 10 \%$ for Brittany and $S_{c_{u}} \simeq 3 \%$ for PACA). The only other technological parameter that may have some influence on the resource estimation is the proportion of installed capacity with dual-axis tracking systems in the technology mix $w_{t_{2}}\left(S_{w_{t_{2}}} \simeq 5 \%\right.$ for Brittany, only about $1 \%$ for PACA).

\section{Conclusion}

Potential assessment generally depends on numerous parameters whose influence on resource estimation relies on complex nonlinear relationships. Conducting a sensitivity analysis may help identify the parameters whose variation has the greatest impact on power potential estimation. In this paper, we proposed a sensitivity analysis methodology based on Sobol indices. We demonstrated the usefulness of our approach through analyzing sensitivity in the technical potential assessment of onshore wind and ground PV resources at regional scale. We took the example of two French regions.

In general, the absence of parameter interaction revealed from the considered model analysis made it easier to identify the most important aspects in determining wind and solar power resource variability. In particular, our results showed that parameters involved in defining surface availability were more prevalent than parameters characterizing technology. We estimated between $65 \%$ and $95 \%$ the relative influence of the former on output estimation variability (65-75\% for onshore wind power and 85-95\% for solar PV). These results bring measurable evidence about which parameters technical potential of onshore wind power and solar PV most depend on, in general.

Furthermore, on a particular case study, the proposed methodology allows to determine precisely which parameters have to be considered, so that efforts prioretization in alleviating associated constraints may result in most increasing the exploitable potential. For instance, results of the present study showed that careful attention should be paid to production capacity density at power plant scale to ensure satisfying onshore wind power potential in Brittany.

To extend this work, a similar analysis could be carried out using additional parameters (e.g. the minimum distance between wind farms and dwellings, foreseen growth of urban territories, or other changes in land use, etc.). It could also include updated analysis as more accurate estimations of input parameters become available, resulting in improved parametric assumptions of associated uncertainty. Finally, the high level of uncertainty associated with potential assessment that this study demonstrates should encourage its systematic investigation in future research.

\section{Acknowledgements}

This work is part of the "Un mix électrique $100 \%$ renouvelable? Analyses et optimisations" study and is supported by the Agence de l'Environnement et de la Maîtrise de l'Energie (ADEME). We thank Réseau de Transport d'Electricité (RTE) and Transvalor for providing part of the data. We also thank Solais for its help and expertise. Finally, we would like to thank the anonymous referees who reviewed this paper for their comments and suggestions.

\section{References}

[1] C. L. Archer and M. Z. Jacobson. Evaluation of global wind power. Journal of Geophysical Research: Atmospheres, 110(D12):n/a-n/a, 2005.

[2] X. Lu, M. B. McElroy, and J. Kiviluoma. Global potential for wind-generated electricity. Proceedings of the National Academy of Sciences, 106(27):10933-10938, 2009.

[3] P. R. Defaix, W. G. J. H. M. van Sark, E. Worrell, and E. de Visser. Technical potential for photovoltaics on buildings in the EU-27. Solar Energy, 86(9):2644 - 2653, 2012. 
[4] P. Moriarty and D. Honnery. What is the global potential for renewable energy? Renewable and Sustainable Energy Reviews, 16(1):244 - 252, 2012.

[5] B. J. M. de Vries, D. P. van Vuuren, and M. M. Hoogwijk. Renewable energy sources: Their global potential for the first-half of the 21st century at a global level: An integrated approach. Energy Policy, 35(4):2590 - 2610, 2007.

[6] J.-F. Mercure and P. Salas. An assessement of global energy resource economic potentials. Energy, 46(1):322 - 336, 2012. Energy and Exergy Modelling of Advance Energy Systems.

[7] W. Kempton, C. L. Archer, A. Dhanju, R. W. Garvine, and M. Z. Jacobson. Large $\mathrm{CO}_{2}$ reductions via offshore wind power matched to inherent storage in energy end-uses. Geophysical Research Letters, 2007.

[8] J. A. Jakubiec and C. F. Reinhart. A method for predicting city-wide electricity gains from photovoltaic panels based on LiDAR and GIS data combined with hourly Daysim simulations. Solar Energy, 93(0):127 - 143, 2013.

[9] N. Lukac, D. laus, S. Seme, B. alik, and G. tumberger. Rating of roofs surfaces regarding their solar potential and suitability for PV systems, based on LiDAR data. Applied Energy, 102(0):803 - 812, 2013.

[10] M. Beccali, P. Columba, V. DAlberti, and V. Franzitta. Assessment of bioenergy potential in Sicily: A GIS-based support methodology. Biomass and Bioenergy, 33(1):79 - 87, 2009.

[11] A. Lopez, B. Roberts, D. Heimiller, N. Blair, and G. Porro. U.S. renewable energy technical potentials: A GIS-based analysis. Technical report, National Renewable Energy Laboratory, 2012.

[12] S. Grassi, N. Chokani, and R. S. Abhari. Large scale technical and economical assessment of wind energy potential with a GIS tool: Case study Iowa. Energy Policy, 45(0):73 - 85, 2012.

[13] T. Hong, C. Koo, J. Park, and H. S. Park. A GIS (geographic information system)-based optimization model for estimating the electricity generation of the rooftop PV (photovoltaic) system. Energy, 65(0):190 - 199, 2014.

[14] S. Izquierdo, M. Rodrigues, and N. Fueyo. A method for estimating the geographical distribution of the available roof surface area for large-scale photovoltaic energy-potential evaluations. Solar Energy, 82(10):929 - 939, 2008.

[15] D. Mac Kay. Could energy intensive industries be powered by carbon-free electricity? Phil Trans R Soc A 371: 20110560, 2013.

[16] S.-D. Kwon. Uncertainty analysis of wind energy potential assessment. Applied Energy, 87(3):856 $865,2010$.

[17] S. Jung, O. A. Vanli, and S.-D. Kwon. Wind energy potential assessment considering the uncertainties due to limited data. Applied Energy, 102(0):1492 - 1503, 2013.

[18] Sensitivity analysis in the technical potential assessment of onshore wind and ground solar photovoltaic power resources at regional scale - Supplementary material.

[19] A. Costa, A. Crespo, J. Navarro, G. Lizcano, H. Madsen, and E. Feitosa. A review on the young history of the wind power short-term prediction. Renewable and Sustainable Energy Reviews, 12(6):1725 $-1744,2008$.

[20] N. Boccard. Capacity factor of wind power realized values vs. estimates. Energy Policy, 37(7):2679 - 2688, 2009. 
[21] B. Chabot. Bright economic and strategic perspectives for onshore wind power in medium to low wind speed areas. WindTech International, Volume 9, N 6 , September 2013.

[22] Le dveloppement de l'olien en Allemagne. Technical report, Fraunhofer IWES, 2013.

[23] P. Blanc, B. Gschwind, M. Lefvre, and L. Wald. The HelioClim project: surface solar irradiance data for climate applications. Remote Sensing, 3(2):343-361, 2011.

[24] T. Muneer. Solar radiation and daylight models for the energy efficient design of buildings. Architectural Press, 1997.

[25] J. A. Ruiz-Arias, H. Alsamamra, J. Tovar-Pescador, and Pozo-Vzquez D. Proposal of a regressive model for the hourly diffuse solar radiation under all sky conditions. Energy Conversion and Management, 51(5):881 - 893, 2010.

[26] Siener, I. and Knoll, B. "Still more than enough". Photon international, february 2013, p.73.

[27] Cap 100\% EnR 2050: Simulation de la production renouvelable et valuation des gisements. Technical report, Agence Departementale pour l'Environnement et la Matrise de l'Energie, 2015.

[28] R. Girard, K. Laquaine, and G. Kariniotakis. Assessment of wind power predictability as a decision factor in the investment phase of wind farms. Applied Energy, 101(0):609 - 617, 2013. Sustainable Development of Energy, Water and Environment Systems.

[29] I.M. Sobol. Global sensitivity indices for nonlinear mathematical models and their Monte Carlo estimates. Mathematics and Computers in Simulation, 55(1-3):271 - 280, 2001. The Second IMACS Seminar on Monte Carlo Methods.

[30] T. Homma and A. Saltelli. Importance measures in global sensitivity analysis of nonlinear models. Reliability Engineering E3 System Safety, 52(1):1 - 17, 1996.

[31] T. Hastie, R. Tibshirani, and J. Friedman. The elements of statistical learning, second edition: Data mining, inference, and prediction. Springer Series in Statistics. Springer, 2nd ed. 2009. corr. 3rd printing edition, 2009.

[32] M. Hoogwijk, B. Vries, and W. Turkenburg. Assessment of the global and regional geographical, technical and economic potential of onshore wind energy. Energy Economics, 26(5):889 - 919, 2004.

[33] EEA. Europe's onshore and offshore wind energy potential. An assessment of environmental and economic constraints. Technical report, European Environment Agency, 2009. 